\title{
Talla de mujeres adultas gestantes en muestras de los sistemas de salud público y privado de Chile
}

\author{
Francisco Mardones $S^{1}$, José Luis Tapia $I^{2}$, Roberto Mallea $A^{a}$, \\ Luis Villarroel del $\mathbf{P}^{\mathbf{1}}$.
Height of pregnant women attended in the public and private health systems in Chile

\begin{abstract}
Background: Adult height has increased around the world, especially in developed countries, probably due to the improvement in life conditions. Aim: To assess differences in women's height in two population samples of different socioeconomic status coming from the public and the private health system in Chile, and the influence of the year of birth on height. Material and methods: Data from 5,416 pregnant women attended in the public health system and 1,658 women attended in the private system, were analyzed. Among women attended in the private system, the association of maternal height with anthropometric indices of the newborn were also studied. Results: Mean height difference in the two systems reached $3,78 \mathrm{~cm}$. The correlation coefficient of maternal height and year of birth was significant although weak in the two health systems, but mean height differences between decades of birth were significant only in the public sector: 0.7 and $1 \mathrm{~cm}$ in each of the two most recent decades. Association of maternal height was significant although weak, with both weight and height at birth, controlling for newborn's sex. Conclusions: Socioeconomic factors influence maternal height. Maternal height has an influence on fetal growth (Rev Méd Chile 2004; 132: 1483-8).
\end{abstract}

(Key Words: Body height; Pregnancy; Socioeconomic factors)

\begin{abstract}
Recibido el 30 de enero, 2004. Aceptado en versión corregida el 6 de octubre, 2004.
${ }_{1}^{1}$ Departamento de Salud Pública, Facultad de Medicina, Pontificia Universidad Católica de Chile.

${ }^{2}$ Departamento de Pediatría, Facultad de Medicina, Pontificia Universidad Católica de Chile.

aAlumno de Cuarto Año de Medicina, Pontificia Universidad Católica de Chile.

bEstadístico.
\end{abstract}

$\mathrm{D}$ esde mediados del siglo XIX, se ha registrado, a escala mundial, un aumento de la talla promedio del adulto, en ambos sexos y en los distintos grupos socioeconómicos. En el norte de Europa y en los Estados Unidos de Norteamérica el incremento de talla del adulto, entre $1880 \mathrm{y}$ 1950, ha sido de más de $1 \mathrm{~cm}$ por década ${ }^{1}$.

Correspondencia a: Dr. Francisco Mardones S. Departamento de Salud Pública, Facultad de Medicina, Pontificia Universidad Católica de Chile. Marcoleta 434, Santiago. Teléfonos: 3546898, 3543038. Fax: 6331840.

E-mail: mardones@med.puc.cl, fmardons@puc.cl
Se ha propuesto que esta tendencia, llamada secular, se debe a mejoras en las condiciones ambientales, especialmente la nutrición. Los factores ambientales parecen ser más relevantes que los genéticos en las diferencias en talla de poblaciones ${ }^{2,3}$. En concordancia con lo anterior, se ha reportado que la talla materna en los países desarrollados es mayor que en aquellos en desarrollo, reflejando las influencias de las mejores condiciones de vida sobre el crecimiento ${ }^{4}$.

Los datos de talla en mujeres adultas chilenas son escasos. En 1960 se realizó el primer estudio del 
estado nutricional de la población chilena y se reportó un promedio de 150,6 cm en una muestra no representativa de 2.078 mujeres de entre 20 y 44 años, de nivel socioeconómico bajo o medio, provenientes de 17 ciudades $^{5}$. Los artículos publicados en los últimos quince años, y con información de la talla en mujeres embarazadas beneficiarias del sistema público de salud, señalan promedios entre 153,5 y 155 $\mathrm{cm}^{6-10}$. La información disponible sobre los resultados de la Encuesta Nacional de Salud (ENS) del año 2003, realizada en una muestra representativa de la población mayor de 17 años de todo el país, muestra un promedio de talla en mujeres de $155,6 \mathrm{~cm}^{11}$.

El primer propósito de este estudio fue conocer los promedios de talla en muestras de mujeres embarazadas atendidas en los sistemas de salud público y privado. Luego, se intentó identificar posibles diferencias de talla entre cohortes de mujeres embarazadas nacidas en diferentes décadas de la segunda mitad del siglo XX en ambos sistemas. Un propósito adicional, que sólo pudo llevarse a cabo con los datos disponibles del sistema privado, fue estudiar la posible asociación de la talla materna con el peso y la talla del recién nacido, considerando la influencia del sexo.

\section{MATERIAL Y MÉTODOS}

En el sistema público de salud, se obtuvo una muestra de embarazadas bajo control proveniente de los consultorios de 23 comunas. Se indican a continuación las seis provincias a las que pertenecen con el nombre de las comunas entre paréntesis: Copiapó (Copiapó), Los Andes (Los Andes, Calle Larga, Rinconada y San Esteban), San Felipe de Aconcagua (San Felipe, Catemu, Llaillay, Panquehue, Putaendo y Santa María), Cordillera (Puente Alto), Concepción (Chiguayante, Concepción, Coronel, Hualqui, Lota, Penco, San Pedro de la Paz, Talcahuano y Tomé), Arauco (Lebu y Cañete).

El 68,32\% de la población chilena es beneficiaria del seguro público o Fondo Nacional de Salud (FONASA) ${ }^{12}$. El resto utiliza el seguro privado o Instituciones de Salud Previsional (ISAPRE), o el sistema previsional de las Fuerzas Armadas.

La muestra del sistema privado se obtuvo de la Maternidad del Hospital Clínico de la Pontificia Universidad Católica (PUC) de Chile, en la que habitualmente más de $70 \%$ de las mujeres atendi- das son beneficiarias de ISAPRE, siendo el resto principalmente de FONASA libre elección. Estos registros incluyen los datos de peso y talla al nacer.

Estas muestras no fueron escogidas al azar dentro de cada universo de población, por lo que no son representativas de toda la población atendida por cada sistema. Las poblaciones respectivas corresponden a todas las mujeres atendidas en los períodos que se indican. Provincia de Copiapó: primeros cinco meses de 2003. Provincias de Los Andes y San Felipe de Aconcagua: primer semestre de 2001. Provincia Cordillera: primeros ocho meses de 2001. Provincia de Concepción: primeros tres meses de 2003. Provincia de Arauco: segundo semestre de 2002. Hospital Clínico PUC: marzo de 2002 a marzo de 2003.

La talla materna se determinó con un cartabón estandarizado con la mujer descalza durante los controles prenatales regulares, registrándose en las fichas maternales respectivas, tanto en la atención primaria como en las fichas de parto.

Se recolectaron sus valores individuales por personal profesional de salud para constituir las bases de datos del sistema público y privado. De la misma manera, se registraron la edad al control de salud o la fecha de nacimiento, dependiendo de la que estuviera presente. En la maternidad de la PUC, la información de peso al nacer se registró en balanzas de palanca y la de talla al nacer con un pediómetro.

Se calcularon los promedios de talla y de edad en mujeres embarazadas adultas de 20 años o mayores para las dos muestras en estudio, considerando que la gran mayoría de las mujeres completan su desarrollo antes de dicha edad ${ }^{13,14}$. La significación estadística de sus diferencias se calculó con la prueba t de Student.

Para analizar los posibles cambios de la talla en el tiempo, se agruparon las mujeres en cohortes o categorías nacidas en diferentes décadas: 1954-1963, 1964-1973 y 1974-1983. Con el dato de edad al momento de consultar o la fecha de nacimiento, se determinó el año de nacimiento de las mujeres de ambas muestras. La significación estadística de las diferencias en los promedios de talla de estos grupos se calculó con el análisis de varianza para un factor y el test de comparaciones múltiples de Bonferroni.

Se estudió la asociación entre la talla y el año de nacimiento de la madre en ambos sistemas usando el coeficiente de correlación $r$ de Pearson. 
Tabla 1. Promedios de talla y edad en las muestras del sistema público y el privado

\begin{tabular}{|c|c|c|c|c|c|}
\hline \multirow[t]{2}{*}{ Sistema } & \multirow[t]{2}{*}{$\mathrm{n}$} & \multicolumn{2}{|c|}{ Talla $(\mathrm{cm})$} & \multicolumn{2}{|c|}{ Edad (años) } \\
\hline & & Promedio & DS & Promedio & DS \\
\hline Público & 5.416 & 156,316 & 6,089 & 28,209 & 5,952 \\
\hline Privado & 1.658 & $160,095 *$ & 6,538 & $30,524^{*}$ & 5,079 \\
\hline
\end{tabular}

*La diferencia con el sistema público fue significativa $(p<0,05)$.

En el sistema privado fue posible estudiar también la asociación conjunta de la talla materna y el sexo del recién nacido con el peso y la talla de nacimiento, utilizando el método de regresión lineal múltiple con el procedimiento paso a paso.

Se consideraron estadísticamente significativos los valores de $\mathrm{p}$ menores de 0,05.

\section{RESULTADOS}

Los estadígrafos de talla y edad en las muestras de ambos sistemas de salud se presentan en la Tabla 1. La diferencia entre los promedios de talla fue de $3,8 \mathrm{~cm}$ a favor de las mujeres atendidas en el sistema privado. También la edad fue mayor en el sistema privado.

Las Tablas 2 y 3 muestran los promedios de talla en las muestras respectivas según categorías de edad y la significación estadística de sus diferencias. En la muestra del sistema privado ninguna diferencia fue significativa.

Los coeficientes de correlación $r$ de Pearson entre talla y año de nacimiento de la madre fueron 0,110 para la muestra del sistema público y 0,061 para el sistema privado, con valores $\mathrm{p}$ de $0,000 \mathrm{y}$ 0,013 , respectivamente.

Los promedios de talla y peso de los recién nacidos de cada sexo de la muestra del sistema privado se muestran en la Tabla 4. Sus diferencias según sexo fueron estadísticamente significativas. Ambos modelos de regresión mostraron que el efecto combinado de la talla y el sexo fue estadísticamente significativo (Tablas 5 y 6).
Tabla 2. Promedios de talla en el sistema público, según año de nacimiento materno

\begin{tabular}{|lrll|}
\hline \multirow{2}{*}{$\begin{array}{l}\text { Categoría de año } \\
\text { de nacimiento }\end{array}$} & $\mathrm{n}$ & \multicolumn{2}{c|}{ Talla (cm) } \\
& & Promedio & DS \\
\hline $1954-1963$ & 327 & $154,936^{*}$ & 6,761 \\
$1964-1973$ & 2.083 & $155,952^{*}$ & 6,072 \\
$1974-1983$ & 3.006 & $156,717 *$ & 6,018 \\
& & & \\
\hline
\end{tabular}

*Las diferencias con todas las otras categorías fueron significativas $(p<0,05)$.

Tabla 3. Promedios de talla en el sistema privado, según año de nacimiento materno

\begin{tabular}{|lrrr|}
\hline $\begin{array}{l}\text { Categoría de año } \\
\text { de nacimiento }\end{array}$ & $\mathrm{n}$ & \multicolumn{2}{c|}{ Talla (cm) } \\
& & Promedio & DS \\
\hline $1954-1963$ & 110 & 158,8180 & 6,755 \\
$1964-1973$ & 932 & 160,130 & 6,446 \\
$1974-1983$ & 611 & 160,272 & 6,622 \\
\hline
\end{tabular}

\section{Discusión}

La talla de estas mujeres adultas tuvo promedios significativamente diferentes entre las muestras del sistema público y el privado. Aunque estas muestras no son representativas de toda la población atendida por cada sistema, ellas abarcan a toda la población atendida en los respectivos servicios de salud y en el Hospital Clínico de la PUC en ciertos períodos de tiempo; todos ellos pueden considerarse de importante cobertura poblacional.

Tabla 4. Promedios de peso y talla al nacer en el sistema privado, según sexo

\begin{tabular}{|llrrrl|}
\hline Sexo & $\mathrm{n}$ & \multicolumn{2}{c}{ Peso RN } & \multicolumn{2}{c|}{ Talla RN } \\
& & Promedio & DS & Promedio & DS \\
\hline Mujeres & 791 & $3.229,03$ & 513,44 & 48,85 & 2,44 \\
Hombres & 871 & $3.324,72^{*}$ & 560,95 & $49,95^{*}$ & 2,99 \\
\hline
\end{tabular}

* La diferencia con el otro sexo fue significativa. 
Tabla 5. M odelo de regresión lineal paso a paso para peso de nacimiento

\begin{tabular}{|lccccc|}
\hline & $\begin{array}{c}\text { Coeficiente } \\
\text { Beta }\end{array}$ & $\begin{array}{c}\text { Error } \\
\text { estándar }\end{array}$ & $\mathrm{p}$ & $\begin{array}{c}\mathrm{R}^{2} \\
\text { parcial }\end{array}$ & $\begin{array}{c}\mathrm{R}^{2} \text { del } \\
\text { modelo }\end{array}$ \\
\hline Talla matema & $1.369,528$ & 203,541 & 0,000 & 0,026 & \\
Sexo & 94,501 & 26,121 & 0,000 & 0,007 & 0,033 \\
Intercepto & $1.036,871$ & 326,349 & 0,002 & & \\
\hline
\end{tabular}

Tabla 6. M odelo de regresión lineal paso a paso para talla de nacimiento

\begin{tabular}{|lccccc|}
\hline & $\begin{array}{c}\text { Coeficiente } \\
\text { Beta }\end{array}$ & $\begin{array}{c}\text { Error } \\
\text { estándar }\end{array}$ & $p$ & $\begin{array}{c}\mathrm{R}^{2} \\
\text { parcial }\end{array}$ & $\begin{array}{c}\mathrm{R}^{2} \text { del } \\
\text { modelo }\end{array}$ \\
\hline Talla materna & 7,926 & 1,033 & 0,000 & 0,034 & \\
Sexo & 0,629 & 0,133 & 0,000 & 0,012 & 0,046 \\
Intercepto & 36,168 & 1,657 & 0,000 & & \\
\hline
\end{tabular}

La diferencia observada en la talla de ambos grupos fue de magnitud relativamente considerable $(3,78 \mathrm{~cm})$, lo que podría estar reflejando la diversidad en los factores ambientales a los que estuvieron expuestas durante su desarrollo. Estas diferencias podrían estar relacionadas, entre otros factores ambientales, con su nivel de ingreso económico, que es más alto en los grupos atendidos por el sistema privado, afiliados en su mayoría a las ISAPRE y a FONASA en sus niveles de mayor ingreso ( $\mathrm{C}$ y $\mathrm{D})$, como se señaló anteriormente. En cambio, los grupos atendidos por el sistema público pertenecen en su mayoría a FONASA en sus niveles de menor ingreso ( $\mathrm{A} \mathrm{y} \mathrm{B}$ ), que no acceden normalmente a la medicina de libre elección. Estas afirmaciones se derivan de los límites de ingreso de las categorías de FONASA ${ }^{12}$.

En este estudio no se incluyeron variables relacionadas con las características étnicas de las mujeres, por lo que no se puede descartar la influencia de este factor en las diferencias.

La diferencia antes mencionada, permite suponer que la mayoría de las mujeres permanece en condiciones semejantes a las de su infancia, lo que está apoyado en las importantes diferencias en talla de niños de distinto nivel socioeconómico observadas en Chile ${ }^{15,16}$. Por ejemplo, preescolares de 5 años de edad estudiados en el año 1965, mostraron una diferencia promedio de $4,5 \mathrm{~cm}$ en grupos socioeconómicos similares a los que se presentan en este estudio ${ }^{17}$. Sin embargo, no se puede descartar que alguna proporción haya cambiado de nivel socioeconómico durante el transcurso de su vida.

Las diferencias de talla parecen estar mucho más asociadas a factores ambientales que genéti$\cos ^{2}$. Se han descrito diferencias porcentuales en talla y peso mucho mayores al comparar poblaciones de preescolares con diferencias socioeconómicas y no étnicas (3\%) que al hacerlo con poblaciones de similar nivel socioeconómico y diferente origen étnico (12\%). La nutrición inadecuada y las enfermedades infecciosas han sido frecuentemente mencionados como los factores ambientales más importantes en las diferencias de crecimiento en niños de diferentes situaciones socioeconómicas. Otro antecedente a favor de esta hipótesis es que la talla materna en los países desarrollados es mayor que en aquellos en desarrollo, reflejando probablemente las mejores influencias ambientales sobre el crecimiento en las niñas ${ }^{18}$.

La tendencia secular hacia una mayor velocidad de crecimiento y una adolescencia más temprana se ha registrado en Europa y América desde mediados del siglo XIX. Esta tendencia apoya la importante influencia de las condiciones ambientales. Implica un crecimiento más precoz en etapas tempranas de la infancia y un aumento de la talla definitiva del adulto. La magnitud de los cambios es considerable. Los registros históricos de Gran Bretaña, países escandinavos y Estados Unidos de Norteamérica mostraron variaciones 
similares entre sí, con ganancias promedio entre 1880 y 1950 de cerca de media pulgada $(1,27 \mathrm{~cm})$ por década para el adulto completamente desarrollado ${ }^{1}$.

Se pudo estimar la influencia del año de nacimiento en la talla. Dado el carácter de las muestras, que consideraron sólo mujeres en edad fértil, el rango de años de nacimiento no fue muy amplio.

La comparación de los cambios en los promedios por década, muestra la existencia de un aumento de talla en el sistema público. Las diferencias entre todas las categorías de año de nacimiento en dicho sistema fueron estadísticamente significativas. Sin embargo, las ganancias por década observadas fueron de 0,77 y $1,02 \mathrm{~cm}$ (Tabla 2), menores que en los países desarrollados, posiblemente porque la mejoría socioeconómica ha sido menos acelerada. En Estados Unidos de Norteamérica datos recientes muestran ganancias medias por década de $1,23 \mathrm{~cm}$ en mujeres blancas y 1,49 en mujeres de origen mexicano ${ }^{19}$, lo que también apoya la idea de una mejoría más pronunciada en los factores ambientales.

Considerando que es muy improbable un cambio en las características genéticas de las poblaciones estudiadas en intervalos de tiempo de esta magnitud, los cambios en el tiempo en la muestra del sector público serían atribuibles a diferencias favorables en la nutrición, acceso a la salud y otros factores ambientales durante el período del crecimiento. Se ha descrito una evolución favorable de los mismos en Chile para los años del estudio ${ }^{20}$. La poca variabilidad de la talla en la muestra del sistema privado podría sugerir que ya está alcanzado su talla máxima determinada genéticamente, o que el tamaño muestral más reducido que en la otra muestra no permitió establecer cambios. A favor del primer argumento está el promedio de talla de mujeres estadounidenses blancas en este mismo rango de edad, que llegó a 163,0 cm entre 1988 y $1994^{19}$. Sin embargo, en los dos sistemas, los coeficientes de correlación entre talla y año de nacimiento fueron significativos pero relativamente bajos, confirmando la menor velocidad de la tendencia secular en Chile.

Aunque existió una diferencia significativa de 2 años en la edad entre los promedios de talla de las muestras, no es esperable que sus diferencias en talla se deban a ello, considerando que la ganancia secular de talla en países desarrollados se ha detectado en $1,27 \mathrm{~cm}$ por década ${ }^{1}$. Sus diferencias en talla se deberían al hecho de provenir de grupos socioeconómicos diferentes.

Los promedios de talla de ambos sistemas de salud fueron superiores a los reportados en artículos recientes ${ }^{6-10}$, y considerablemente mayores a los encontrados en $1960^{5}$. La muestra estudiada aquel año tampoco fue representativa de la población nacional, pero su composición por edad y nivel socioeconómico fue similar a la de la muestra del sistema público del presente estudio. La diferencia de $5,7 \mathrm{~cm}$ con el promedio del estudio actual, realizado poco más de 40 años después, es compatible con la idea de un aumento cercano a $1 \mathrm{~cm}$ por década.

Por otra parte, los promedios de estas muestras, en particular la del sistema público, son levemente superiores a los encontrados en la ENS $^{11}$, lo que sugiere que la aproximación con que reflejan la realidad nacional es aceptable. La diferencia posiblemente se deba a que la ENS incluyó también a población mayor de 50 años. La información disponible, hasta el momento, de la ENS, muestra que los datos de talla agregados de hombres y mujeres también se relacionan en forma negativa con la edad ${ }^{11}$.

Las asociaciones de talla materna con peso y talla de nacimiento fueron significativas, pero los coeficientes $r^{2}$ de los modelos de regresión lineal múltiple resultaron relativamente bajos (Tablas $5 \mathrm{y}$ 6), por lo que su capacidad predictiva fue escasa. Ello es compatible con el conocido efecto de otros factores, como el estado nutricional de la madre y las patologías del embarazo, en el crecimiento fetal ${ }^{21}$.

Sin embargo, la talla de las madres tiene una importante influencia en el cambio de talla entre generaciones ${ }^{22}$. Ello se expresa particularmente en su efecto dominante frente a la talla paterna, que se ha observado en equinos y confirmado epidemiológicamente en huma$\operatorname{nos}^{23}$. Este efecto se atribuye a la influencia del ambiente intrauterino sobre el crecimiento fetal. En Aberdeen, Escocia, se ha observado que los recién nacidos de las madres más bajas y delgadas tienen en promedio cerca de $1 \mathrm{~kg}$ menos de peso que los hijos de aquellas con mayores tallas y pesos ${ }^{23}$. 


\section{REFERENCIAS}

1. TANNER JM. Education and physical growth. London: University of London Press Ltd. 1970.

2. Habicht JP, Martorell $R$, Yarbrough $C$, Malina $R$, KiEIN R. Height and weight standars for preschool children. How relevant are ethnic differences in growth potential? Lancet 1974; 1: 611-4.

3. Monckeberg F, UauY R, CRISTI MA. El chileno está aumentando de estatura. Creces 1984; 5: 17-20.

4. Institute of Medicine, National Academy of Sciences. Nutrition during pregnancy. Washington, DC: National Academy Press, 1990.

5. Interdepartmental Committee on Nutrition for National Defense. Chile, Nutrition Survey (MarchJune 1960). Washington DC: Interdepartmental Committee on Nutrition for National Defense, 1961.

6. MARDones F, Rosso P. Desarrollo de una curva patrón de incrementos ponderales para la embarazada. Rev Méd Chile 1997; 125: 1437-48.

7. Mardones-Santander F, Salazar G, Rosso P, ViliaRROEL L. Maternal body composition near term and birth weight. Obstet Gynecol 1998; 91: 873-7.

8. Durán E, Soto D, Asenjo G, Pradenas F, Quiroz V. Evaluación de la dieta de embarazadas de área urbana y su relación con el estado nutricional. Rev Chil Nutr 1999; 26: 62-9.

9. Mardones-Santander F, Rosso P, Stekel A, Ahumada E, Luaguno S, Pizarro F et al. Effect of a milkbased food supplement on maternal nutritional status fetal growth in underweight Chilean women. Am J Clin Nutr 1988; 47: 413-9.

10. Atalah E, Castilo C, Castro R, Aldea A. Propuesta de un nuevo estándar de evaluación nutricional en embarazadas. Rev Méd Chile 1997; 125: 1429-36.

11. Ministerio de Salud, Chile. Encuesta Nacional de Salud 2003. Sitio web: www.minsal.cl, 2004.

12. FONASA, Departamento de Gestión Estratégica Estudios. Abril, 2003.

13. Rosso P. Aspectos biológicos del desarrollo. En: Meneghello J. ed. Pediatría. Buenos Aires: Editorial Médica Panamericana, 1997; 70-7.

14. NeEDLman R. Growth and Development. En: Behrman R, Kliegman R, Jenson $H$, eds. Nelson Textbook of Pediatrics. Philadelphia: WB. Saunders Company, 2000; 52-7.

15. Muzo S. Crecimiento normal y patológico del niño y del adolescente. Rev Chil Nutr 2003; 30: 92-100.
16. Burrows R, Leiva L, Zvaighaft A, Muzo S. Influencia del nivel socioeconómico (NSE) en la composición corporal y en la estatura de los escolares, durante la pubertad. Rev Chil Nutr 1989; 17: 3945.

17. Barja I, De La Fuente M, Bauester D, Monckeberg F, Donoso G. Peso y talla de preescolares chilenos de tres niveles de vida. Rev Chil Pediatr 1965; 36: 525-9.

18. Krasovec K. Height. Background Issues. En: Krasovec $\mathrm{K}$, Anderson MA, eds. Maternal Nutrition and pregnancy outcomes. Washington DC: Pan American Health Organization, 1991; 93-103.

19. National Center for Health Statistics. Height in centimeters for females 20 years and over-number of examined persons, mean, standard error of the mean, and selected percentiles, by race-ethnicity and age: United States, 1988-1994 [en línea]. Hyattsville MD: National Center for Health Statistics [fecha de consulta: 23 enero 2004]. Disponible en: <http://www.cdc.gov/nchs/>.

20. Meneghelo J, Rosselot J, Mardones RF. El rol de la pediatría clínica y social en los avances de la salud del niño y de la familia, Chile 1900-1995. Rev Pediatría al día 1995; 11: 223-32.

21. Rosso P. Nutrition and metabolism in pregnancy. New York, Oxford University Press, 1990.

22. BARKER DJP. Mother, babies and health in later life. Second ed. Edinburgh, Churchill Livingstone, 1998.

23. Thомson AM. Fetal growth and size at birth. En: Barron SL, Thomson AM, eds. Obstetrical Epidemiology. New York: Academic Press Inc., 1983; 114-6.

\section{Agradecimientos}

A las siguientes personas se les reconoce su valiosa participación en la recolección de datos: Matronas Ángela Zúñiga (Servicio de Salud Arauco), Manola Román (Servicio de Salud Aconcagua) y Jessica Rojas (Servicio de Salud Copiapó), Nutricionista Eliana Durán de la Universidad de Concepción, alumnos de Primer Año de Medicina de 2002 de la Pontificia Universidad Católica de Chile Eugenio Zalaquett, Javier Castro, Felipe Cichero, Ignacio Cifuentes, Cristian Díaz, Cristian González, Daniel Helguero, Juan Huidobro, Víctor Luengo, Cristian Morales y Luis Zavala. 